Research Article

\title{
An Investigation into the Modeling Methodology of the Coil Spring
}

\author{
Liangcheng Dai, Maoru Chi $(\mathbb{D}$, Hongxing Gao, and Jianfeng Sun \\ State Key Laboratory of Traction Power, Southwest Jiaotong University, Chengdu 610031, China \\ Correspondence should be addressed to Maoru Chi; cmr2000@163.com
}

Received 9 May 2020; Revised 5 June 2020; Accepted 6 June 2020; Published 24 June 2020

Academic Editor: Mahdi Mohammadpour

Copyright (C) 2020 Liangcheng Dai et al. This is an open access article distributed under the Creative Commons Attribution License, which permits unrestricted use, distribution, and reproduction in any medium, provided the original work is properly cited.

\begin{abstract}
The coil spring is an important element in the suspension system of railway vehicles, and its structural vibration caused by the mass distribution can deteriorate the dynamic performance of the vehicle. However, the coil spring is usually modelled as a simple linear force element without considering the dynamic characteristics in multibody dynamic simulations of railway vehicles. To integrate the dynamic characteristics of the coil spring into the simulation, three equivalent dynamic models of the coil spring are established by treating the coil spring as multimass spring series, Timoshenko beam, and flexible spring, respectively. The frequency-sweep method is applied to obtain the dynamic response of the proposed models of coil spring, and the accuracy of the models' results has been compared and verified by the laboratory test. Results show that all of these three equivalent models can reflect the influence of the spring mass distribution on its dynamic responses. Compared with the mass-spring series and beam element equivalent models, the flexible spring model can better reflect the dynamic stiffness and stress of the coil spring changing with the exciting frequency. Thus, the flexible spring model proposed in this paper is more applicable to railway vehicle system dynamics and the fatigue analysis.
\end{abstract}

\section{Introduction}

For modern railway vehicles, multibody vehicle dynamics simulation has become a very common and important design instrument, allowing the assessment and optimization of vehicle performance from the early stage of the design process. The accuracy of rail vehicle multibody models is mainly affected by the wheel-rail contact model and the suspension component model [1]. The suspension system mainly consists of the coil spring, rubber spring, air spring, and hydraulic damper. In the previous multibody dynamics analysis of railway vehicles, most suspension components are considered as linear force elements. The models of the air spring, including the linear Nishimura model, spring and dash model, Berg model, thermodynamic model, and TPL-ASN model, have been established in recent years $[2,3]$. For the rubber spring, the one-dimensional model, multidimensional model, and friction model have been established [4]. Nevertheless, it is hard to establish the accurate model for the rubber spring because of its significant nonlinear characteristics, and the neural network model starting from the experimental results has been exploited for vehicle dynamics simulations. In terms of the hydraulic damper, the Maxwell model, consisting of a linear spring in series with a linear dashpot, is widely used in railway vehicle dynamics and has been integrated into the simulation software (SIMPACK, ADAMS/RAIL, etc.) $[5,6]$.

In terms of the coil spring, the stiffness will change with the exciting frequency due to its dynamic characteristics determined by the mass distribution of the spring itself [7]. As the random track irregularities cover the entire frequency spectrum, the structural modes of the coil spring can be excited at a specific frequency from the track excitation, which can significantly change the stiffness of the coil spring and leads to the deterioration of the vehicle performance and spring fatigue life [8, 9]. Zhou et al. [10] studied the failure phenomenon of primary springs for the metro vehicle and found that an obvious resonance phenomenon of the coil spring exists. The field test and finite element analysis were carried out to obtain the modal frequency of the spring under the constraint state. Lee and Thompson [7] used the dynamic stiffness matrix approach 
to obtain the natural frequencies and dynamic stiffness of coil springs. The results exhibit the significant dynamic stiffening effect on coil springs. Combined with the deformation characteristics of the slender spring structure, Zhang et al. [11] studied the nonlinear characteristics of the stiffness of the coil spring with circular section on the basis of the accurate spring beam element model. Sun et al. [12, 13] established the finite element model of the coil spring and analyzed the dynamic stiffness characteristics of the spring by using the mode superposition method. The spring exhibits a strong internal resonance at the frequency around $50-60 \mathrm{~Hz}$, and large stresses arise. Fu and Wang [14] simulated the high frequency vibration of vehicle with coil spring suspension using shock wave theory. Liu and Zhang [15] established the finite element model of the central suspension spring and proposed the method of multidegree of freedom equivalent spring model to obtain the dynamic stiffness. Most studies about fatigue breakage of coil springs focused on material and processing procedures $[16,17]$, while the measures to increase the service life of the coil spring have not been given enough attention. Furthermore, the origin of the occurrence of the high stresses should be clarified, especially for the phenomenon of resonance. However, the dynamic stress of the coil spring in the case of resonance has been rarely studied in multibody dynamic simulations.

The numerical method of dynamic stiffness can only get the approximate solution of coil spring [12, 18-21], while the finite element model of the coil spring can obtain the accurate solution under the premise of sacrificing computing efficiency [22]. In order to consider the changes of stiffness and stress with frequency caused by spring mass and vibration in the multibody vehicle dynamics simulation, three equivalent dynamic models of coil spring are established by using, respectively, three methods of mass-spring series, Timoshenko beam, and flexible body substructure. Then the frequency domain characteristics of coil spring are introduced into time domain integral calculation. In the multi mass-spring equivalent model, to consider the mass and self-vibration of the spring, the continuous coil spring is discretized into several mass blocks. The Timoshenko-beam equivalent model is based on the Timoshenko beam theory and the dynamic stiffness matrix to discretize the coil spring into beam elements. The mass and geometry of the spring are considered in the beam element properties. The equivalent dynamic model of flexible spring is obtained by reducing the degree of freedom of the finite element model, which includes material properties and modal information. In addition, the dynamic stress of flexible spring is obtained by modal stress recovery method. The dynamic response of coil spring under different frequencies is obtained by loading sweep excitation on three equivalent models. Finally, the dynamic characteristics of the spring and the accuracy of the three equivalent models are verified by the dynamic characteristics test.

\section{Equivalent Dynamic Model of Coil Spring}

In the previous multibody dynamics analysis of vehicles, the coil spring is considered as a linear force element. Due to the mass distribution of the spring and the characteristics of selfvibration, the stiffness of the spring changes with frequency. Especially, when the frequency is high or in the resonance region, the change of stiffness is very significant. However, the excitation frequency of the coil spring distributes in a wide range during the vehicle operation. In order to analyze the variation of the dynamic stiffness of a coil spring with the exciting frequency, the following three equivalent spring models have been proposed to present its dynamic characteristics in the multibody dynamics.

2.1. Mass-Spring Series Model. Vehicle dynamic performance simulation is based on time domain. However, the relationship between spring dynamic stiffness and excitation frequency is based on frequency domain. To reflect the frequency-dependent characteristics of spring in time domain, the continuous coil spring is replaced by mass-spring series model with multiple degrees of freedom. The equivalent model is composed of $N-1$ linear supporting force elements and $N$ mass body in series. The number of mass discretization $N$ depends on the order of spring modes or the cut-off frequency of analysis to be reflected in the equivalent model. In order to reflect the self-contact phenomenon of coil spring in abnormal vibration, contact force elements of spring are established between mass bodies, as shown in Figure 1.

The equivalent model consists of several supporting force elements and contact force elements. The support force element is used to connect two continuous mass bodies, which are made up of the linear spring and the damper in parallel. The contact force element of the coil spring is used to connect the mass bodies that may be touched. When these mass bodies contact, the element force provides the corresponding contact force. follows:

The relationship for the support force can be written as

$$
F_{z}=k_{i} \cdot r_{i}+c_{i} \cdot u_{i}
$$

where $F_{z}$ denotes the damping force in $z$ direction, $k_{i}$ $(1 \leq i \leq N-1)$ is the stiffness coefficient, $c_{i}$ is the damping coefficient, $r_{i}$ is the relative displacement between two mass bodies, $u_{i}$ is the relative velocity between two mass bodies, and its calculation formula is as follows:

$$
\left.\begin{array}{l}
r_{i}=\operatorname{st}(i)-\operatorname{st}(i-1)-\Delta z_{0} \\
u_{i}=\operatorname{std}(i)-\operatorname{std}(i-1)
\end{array}\right\},
$$

where $\Delta z_{0}$ is the nominal length of two mass bodies; st ( $i$ ), std ( $i)$ are the state displacement and state velocity of $i$ mass body; st $(i-1)$, std $(i-1)$ are the state displacement and state velocity of $i-1$ mass body.

The relationship for the contact force can be written as follows:

$$
F_{N}=k_{N} p_{i}
$$

where $F_{N}$ denotes the contact force in $z$ direction, $k_{N}$ is the contact stiffness coefficient, and $p_{i}$ is the contact penetration depth. The contact penetration depth is calculated as 


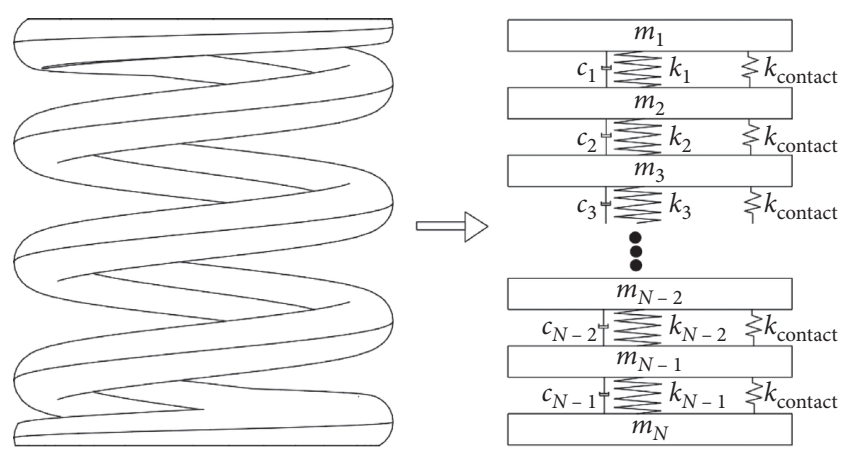

FIGURE 1: Mass-spring series equivalent model.

$$
p_{i}=z(i)-z(i-1)-\left(\Delta z_{o}-L\right)-z_{\mathrm{tol}}
$$

where $z(i)$ is the state displacement of the contact point, and $z(i-1)$ is the state displacement of the contacted point. $\Delta z_{o}$ is the nominal distance between the coils, $L$ is the positive sum of the distances between the respective contact location and associated marker, and $z_{\mathrm{tol}}$ is the adjustment parameter of the contact distance.

In order to ensure that the static stiffness and modal of the equivalent model are equal to the actual spring, the following conditions should be satisfied:

$$
\left.\begin{array}{l}
k_{i}=(N-1) \cdot K_{s} \\
m_{i}=\frac{M_{s}}{N}
\end{array}\right\}
$$

where $K_{s}$ is the static stiffness of the coil spring and $M_{s}$ is the total mass of the coil spring. When the natural frequencies and modes of the equivalent model are calculated, the influence of the damping and contact force elements can be neglected, and the free vibration equation of the spring can be simplified as follows:

$$
M \ddot{x}+K x=0 .
$$

When sinusoidal excitation is applied,

$$
u(t)=A \sin (\omega t+\theta) \text {. }
$$

Substituting equation (7) into equation (8), we obtain

$$
\left(K-\omega^{2} M\right) A \sin (\omega t+\theta)=0 .
$$

In order for equation (8) to have a non-zero solution, it is necessary for the determinant of the matrix $\left[K-\omega^{2} M\right]$ to vanish. The constrained frequency $\omega_{N}$ and movement mode of the multi-mass-spring series model are obtained as shown in Table 1.

The equivalent model is one-dimensional mass-spring series, so each mass body has only vertical degrees of freedom. The spring stiffness is established by linear spring and damping between the springs. The contact force element of the coil spring is used to connect the mass bodies that may be touched. The equivalent spring model can only reflect its own vertical vibration, so the dynamic stiffness calculated is only suitable for one-dimensional model.

2.2. Timoshenko-Beam Model. For the convenience of the spiral spring calculation, the global coordinate system is transformed into the helical curve coordinate system. After the coordinate transformation, the coil spring can be regarded as a simple beam for calculation. The helix radius is $R$ and the helix angle is $\alpha$. The variable $s$ is used to measure the distance along the wire and is related to the angle $\phi$ by

$$
\phi=s \cdot \frac{\cos \alpha}{R} \text {. }
$$

At any point on the helix, local coordinates are defined as shown in Figure 2, with $u$ radial, $w$ tangential, and $v$ binormal to the other directions. The displacements $(u, v, w)$ in these local coordinates are related to those in global coordinates $\left(u_{x}, u_{y}, u_{z}\right)$ by

$$
\left\{\begin{array}{c}
u \\
v \\
w
\end{array}\right\}=\left[\begin{array}{ccc}
0 & -\cos \phi & -\sin \phi \\
\cos \alpha & \sin \alpha \sin \phi & -\sin \alpha \cos \phi \\
\sin \alpha & -\cos \alpha \sin \phi & -\cos \alpha \cos \phi
\end{array}\right]\left\{\begin{array}{l}
u_{x} \\
u_{y} \\
u_{z}
\end{array}\right\}=Q\left\{\begin{array}{l}
u_{x} \\
u_{y} \\
u_{z}
\end{array}\right\} .
$$

Consider the situation in which the spring is subjected to an arbitrary load $F_{1}$. Then at any cross-section the wire is subjected to three components of force $P_{u}, P_{v}, P_{w}$ and three moments $M_{u}, M_{v}, M_{w}$ about the $u, v$ and $w$ directions. These forces and moments result in the linear displacements $\delta$ and rotations $\theta$. According to Timoshenko beam theory, the relationship between load and deformation is as follows [7]:

$$
\frac{\partial}{\partial s}\left\{\begin{array}{c}
\delta \\
\theta \\
P \\
M
\end{array}\right\}=\left[\begin{array}{ccc}
\frac{P_{u}}{G A \gamma}+\theta_{v}+\tau \delta_{v}-\kappa \delta_{w} & \frac{P_{v}}{G A \gamma}-\theta_{u}-\tau \delta_{u} & \frac{P_{w}}{E A}+\kappa \delta_{u} \\
\frac{M_{u}}{E I_{u}}+\tau \theta_{v}-\kappa \theta_{w} & \frac{M_{v}}{E I_{v}}-\tau \theta_{u} & \frac{M_{w}}{G I_{w}}+\kappa \theta_{u} \\
\tau P_{v}-\kappa P_{w} & -\tau P_{u} & \kappa P_{u} \\
P_{v}+\tau M_{v}-\kappa M_{w} & -P_{u}-\tau M_{u} & \kappa M_{u}
\end{array}\right]\left\{\begin{array}{c}
\widehat{u} \\
\widehat{v} \\
\widehat{w}
\end{array}\right\},
$$


TABLE 1: The constrained modes of the coil spring.

\begin{tabular}{|c|c|c|c|c|c|c|c|c|}
\hline Order & 1 & 2 & 3 & 4 & 5 & 6 & 7 & 8 \\
\hline Frequency $(\mathrm{Hz})$ & 65.8 & 129.2 & 187.4 & 237.7 & 247.7 & 277.2 & 301.9 & 302.4 \\
\hline Movement mode & Expansion & Expansion & Expansion & Expansion & Expansion & Expansion & Expansion & Expansion \\
\hline
\end{tabular}

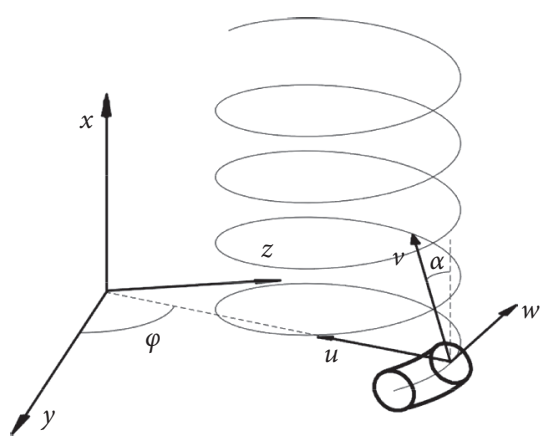

FIgURE 2: Coordinate system of a coil spring.

where $E$ is the Young's modulus, $G$ is the shear modulus, $A$ is the cross-sectional area of the wire, $\gamma$ is the shear area correction, and $I_{u}$ and $I_{v}$ are the second moments of area of the section about the directions $u$ and $v$. Upon rearranging by components of displacements and forces, this can be written as

$$
\frac{\partial}{\partial s}\left\{\begin{array}{c}
\theta \\
P \\
M
\end{array}\right\}=\left[\begin{array}{cc}
S_{11} & S_{12} \\
0 & S_{22}
\end{array}\right]\left\{\begin{array}{c}
\delta \\
\theta \\
P \\
M
\end{array}\right\}
$$

When subjected to dynamic loads, the mass and vibration of the spring need to be considered in the balance equation. Introducing the inertia forces into equation (12) yields the governing partial differential equations for the dynamic equilibrium as

$$
\frac{\partial}{\partial s}\left\{\begin{array}{c}
\theta \\
P \\
M
\end{array}\right\}=\left[\begin{array}{cc}
S_{11} & S_{12} \\
0 & S_{22}
\end{array}\right]\left\{\begin{array}{c}
\delta \\
\theta \\
P \\
M
\end{array}\right\}+\left[\begin{array}{cc}
0 & 0 \\
T_{21} & 0
\end{array}\right] \frac{\partial^{2}}{\partial t^{2}}\left\{\begin{array}{c}
\delta \\
\theta \\
P \\
M
\end{array}\right\},
$$

where $T_{21}$ is a diagonal matrix related to density, crosssectional area, and the second moments of area. The wave in the spring contains spatial and transient variables, which are functions of wave number $k$ and angular frequency $\omega$. If the response is harmonic in time, we write the displacements and forces for a particular free wave as

$$
\left\{\begin{array}{c}
\delta \\
\theta \\
P \\
M
\end{array}\right\}=\left\{\begin{array}{c}
\Delta \\
\Theta \\
\Pi \\
\Lambda
\end{array}\right\} e^{k s+j \omega t}
$$

Substituting equation (14) into equation (15) gives a set of 12 homogeneous linear simultaneous equations:

$$
\left(k[I]-\left[\begin{array}{ll}
S_{11} & S_{12} \\
S_{21} & S_{22}
\end{array}\right]\right)\left\{\begin{array}{c}
\Delta \\
\Theta \\
\Pi \\
\Lambda
\end{array}\right\}=0,
$$

where $S_{21}=-\omega^{2} T_{21}$ and $I$ is the unit matrix. In order for equation (15) to have a non-zero solution, it is necessary for the determinant of the matrix $(k[I]-S)$ to vanish. In order to obtain the point stiffness and transfer stiffness of the coil spring, the force and displacement vectors of the spring are as follows:

$$
\left.\begin{array}{l}
F=\left[\begin{array}{ll}
P(0) & P(L)
\end{array}\right]^{\mathrm{T}} \\
U=\left[\begin{array}{ll}
\delta(0) & \delta(L)
\end{array}\right]^{\mathrm{T}}
\end{array}\right\}
$$

where 0 and $L$ are the two ends of the spring, respectively, and the dynamic stiffness of the spring is defined as

$$
K=F U^{-1} \text {. }
$$

The coil spring is considered as Timoshenko beam for dynamic stiffness matrix analysis. The mass distribution and self-vibration of the coil spring are introduced into the dynamic stiffness analysis, and the relationship between the stiffness of the spring and the frequency is obtained. Based on this theory, the Timoshenko beam equivalent dynamic model of coil spring is proposed. The parameters of the coil spring calculated in this paper are shown in Table 2.

According to the above parameters of the spiral coil spring, the expression under the Cartesian coordinate system of the coil spring is as follows:

$$
\left\{\begin{array}{l}
x_{i}=\frac{D}{2} \cos \left(\frac{2 \pi}{n} i\right) \\
y_{i}=\frac{D}{2} \sin \left(\frac{2 \pi}{n} i\right) ; \quad i=0,1,2 \cdots N \cdot n \\
z_{i}=\frac{S}{n} i
\end{array}\right.
$$

where $n$ is the discrete number of each cycle spring, and the other parameters are shown in Table 2. The Cartesian coordinates of the key points of the center line of the coil spring are obtained by using the helix equation, and the helix is generated according to the key points in the cylindrical coordinates. The helix of the coil spring is shown in Figure 3(a). The continuous coil spring is discretized by beam element, then the discrete element is given material and section properties to ensure that the mass distribution of the beam element model is consistent with the real coil 
TABle 2: Parameters of the coil spring.

\begin{tabular}{lccc}
\hline Notation & Parameter & SI unit & Value \\
\hline$N$ & Total number of cycles & - & 4.6 \\
$n_{1}$ & Number of active coils & - & 3.8 \\
$D$ & Wire diameter & $\mathrm{mm}$ & 40 \\
$D$ & Mean coil diameter & $\mathrm{mm}$ & 240 \\
$\alpha$ & Helical angle & $\mathrm{deg}$ & 5.68 \\
$S$ & Pitch & $\mathrm{mm}$ & 70 \\
$H_{0}$ & Free height & $\mathrm{mm}$ & 315 \\
$G$ & Shear modulus & $\mathrm{N} \cdot \mathrm{mm}^{-2}$ & 78500 \\
$\lambda$ & Poisson's ratio & - & 0.3 \\
\hline
\end{tabular}

spring. Then the dynamic response of the equivalent model is calculated by using Timoshenko beam theory. The equivalent model of beam element is shown in Figure 3(b).

In order to consider the contact nonlinearity of the spring in the equivalent model of beam element, the contact force element is established between two upper and lower nodes on the spring helix. The method of establishing the contact force element refers to Section 2.1.

The lower end of the beam element equivalent model is fixed, the non-zero displacement constraint is applied at the upper surface, and the constraint mode of equivalent model is obtained as shown in Table 3.

In Timoshenko beam equivalent model, structural material properties and spirals are defined by beam element, but the nonuniformity of spring structure is not included. Therefore, all modes of coil spring can be covered. The modal frequencies and shape are basically consistent with the results of finite element mode.

\subsection{Flexible Spring Model}

2.3.1. Dynamic Theory of Flexible Bodies. The model of spring flexible body is mainly based on the fixed interface modal synthesis method [23]. Firstly, a modal set is given to the flexible body, which is expanded by modal method. The elastic displacement of the flexible body is represented by the linear combination of modal vector and modal coordinate. The elastic displacement of the flexible body is calculated at each moment to express its deformation. The interface of flexible components is fixed and the modal matrix is established, then the modal matrix can be expressed as

$$
\varphi_{r}=\left[\varphi_{1 N}, \varphi_{1 C}\right],
$$

where $\varphi_{1 N}$ is the main modal matrix and $\varphi_{1 C}$ is the constrained modal matrix. The main mode $q_{N}$ is the intrinsic mode when the interface degrees of freedom are fixed, which corresponds to the internal degrees of freedom. The constrained mode $q_{C}$ is the static mode formed by the static displacement distribution, which releases each boundary degree of freedom in turn to produce unit displacement. Then the displacement matrix $s$ is as follows:

$$
s=\left[\begin{array}{cc}
I & 0 \\
\varphi_{1 N} & \varphi_{1 C}
\end{array}\right]\left[\begin{array}{l}
q_{C} \\
q_{N}
\end{array}\right],
$$

where $I$ is unit matrices and 0 is zero matrices. Through the transformation, the flexible body equation of motion is obtained:

$$
\widehat{M} \ddot{s}+\widehat{K} s=f(\theta, t),
$$

where $\widehat{M}, \widehat{K}$ are, respectively, transformed mass matrix and stiffness matrix; $f$ is a function of constrained modal force and inherent modal force of flexible body; $\theta$ is a state variable of multibody system; $t$ is a time variable.

2.3.2. Modelling of Flexible Spring. In the multibody dynamics calculation, the equivalent model of the coil spring should not only reflect the spring's own vibration, but also ensure the three-dimensional support stiffness of the spring. Based on the theory of flexible body, a flexible body model of coil spring is established to reflect the dynamic characteristics caused by mass distribution and self-vibration of the spring. The spring flexible model can include both static stiffness and dynamic characteristics. The modeling process is shown in Figure 4.

In order to accurately reflect the characteristics of the coil spring, the geometric model of the spring is established and given the corresponding material properties. Then the geometric model is discretized by the solid element SOLID92 with 6 degrees of freedom. The finite element model of the spring is obtained as shown in Figure 5. The lower surface of the finite element model is fixed, and the non-zero displacement constraint is applied at the upper surface. The constraint mode of the finite element model is obtained as shown in Figure 5.

In order to improve the efficiency of dynamic calculation of coil spring, the finite element model is reduced in degrees of freedom, and the complete spring structure with more degrees of freedom is transformed into a structure with lower degree of freedom by substructure reduction method. The reduced model has the same static and dynamic characteristics as the original structure. In order to retain the stiffness information of springs, the modes within $1000 \mathrm{~Hz}$ are selected as the main modes, and the substructures are generated based on the finite element models. Before the dynamic calculation, the structural damping of flexible spring is defined, and then the constraint mode of flexible spring is obtained. The constrained modes of the finite element model are compared with those of the flexible body as shown in Table 4.

Comparing the constrained modal results of the full degree of freedom spring with that of the flexible spring (substructure), it can be found that the flexible spring includes the structure and modal properties of the spring. The substructure can match the modal frequencies and movement modes with the full degree of freedom spring in the frequency range considered.

2.3.3. Dynamic Stress Calculation of Coil Spring. The traditional rigid-body dynamic model neglects the relative displacement inside the structure. When the structure resonates in the vibration, the calculated stress will be less than the actual structural stress if the traditional static stress calculation method 


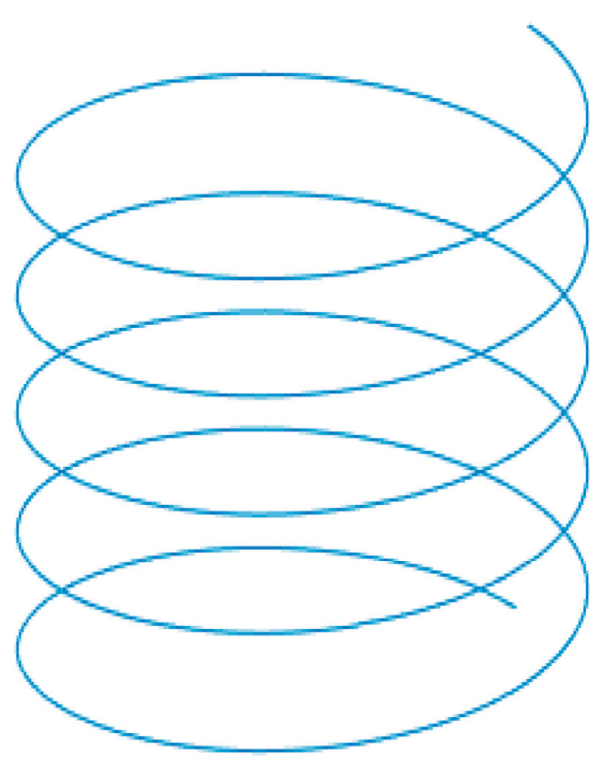

(a)

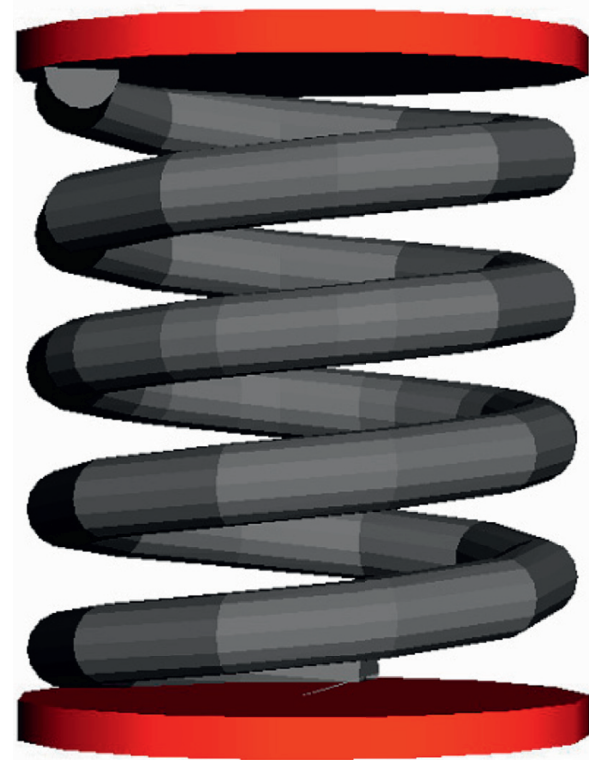

(b)

FIgure 3: Coil spring model. (a) Helix. (b) Beam element equivalent model.

TABle 3: The constrained modes of the coil spring.

\begin{tabular}{|c|c|c|c|c|c|c|c|c|}
\hline Order & 1 & 2 & 3 & 4 & 5 & 6 & 7 & 8 \\
\hline Frequency $(\mathrm{Hz})$ & 66.8 & 71.8 & 84.1 & 88.5 & 122.9 & 127.5 & 137.1 & 144.2 \\
\hline Movement mode & Expansion & Twist & Bending & Bending & Bending & Expansion & Bending & Twist \\
\hline
\end{tabular}

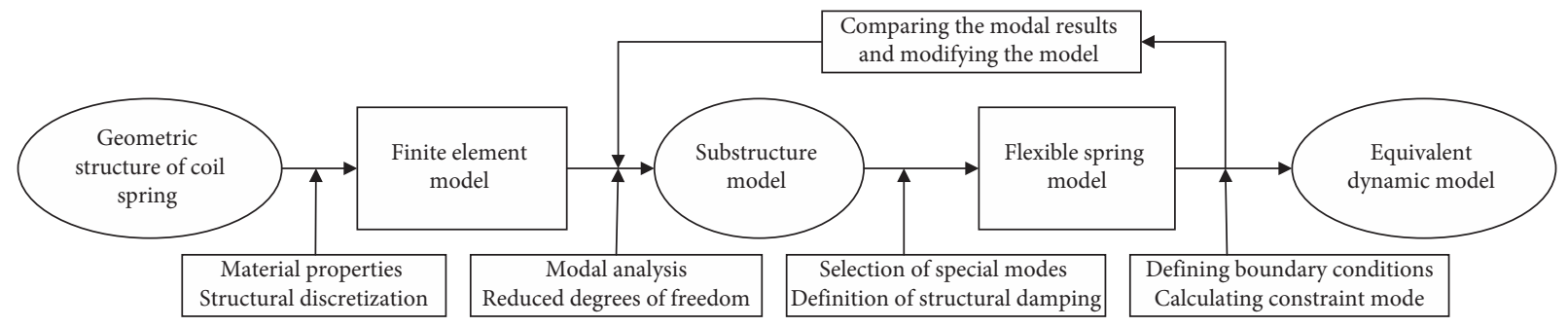

FIgURE 4: Modeling flow of flexible spring.

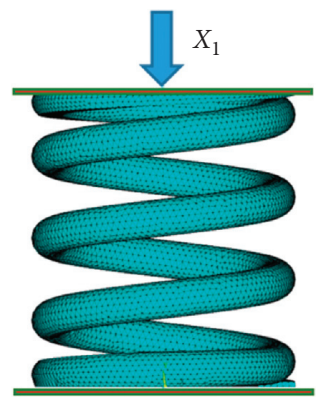

(a)
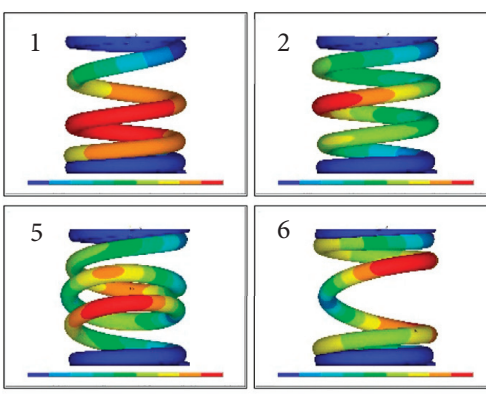

(b)

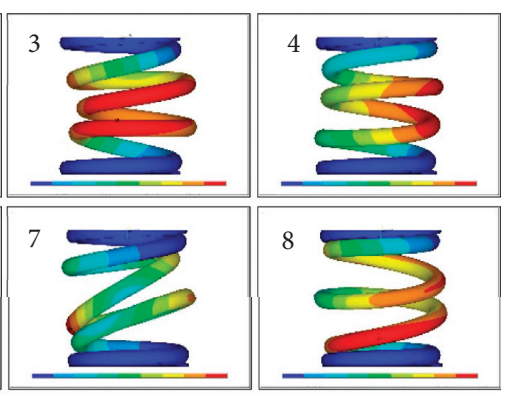

FIgURE 5: The FEM model and constrained modes of the coil spring. 
TABLE 4: The constrained modes of the coil spring.

\begin{tabular}{lcccccccc}
\hline Order & 1 & 2 & 3 & 4 & 5 & 6 & 7 & 8 \\
\hline Finite element model $(\mathrm{Hz})$ & 66.1 & 70.7 & 88.9 & 89.7 & 117.7 & 122.9 & 128.8 & 133.5 \\
Substructure model $(\mathrm{Hz})$ & 66.1 & 70.6 & 88.7 & 89.4 & 117.3 & 122.3 & 128.2 & 132.7 \\
Movement mode & Expansion & Twist & Bending & Bending & Bending & Expansion & Bending & Bending \\
\hline
\end{tabular}

is used [24]. In order to calculate the structural dynamic stress when the coil spring resonates, the structural stress is calculated by modal stress recovery based on the structural modal displacement. Modal displacement $X$ of a node in a flexible body can be obtained by superposition of several modes:

$$
X=\sum_{j=1}^{H} h_{j} w_{j}=H w,
$$

where $h_{j}$ is the mode frequency of the $j$ th order and $w_{j}$ is the mode displacement corresponding to the mode frequency of the $j$ th order. In the calculation of structural finite element method, the strain $\varepsilon_{i}^{e}$ and stress $\sigma_{i}^{e}$ corresponding to node $i$ are as follows:

$$
\left\{\begin{array}{l}
\varepsilon_{i}^{e}=B_{i}^{e}\left(X_{i}^{e}\right) u_{i}^{e}, \\
\sigma_{i}^{e}=D_{i}^{e} \varepsilon_{i}^{e}=D_{i}^{e} B_{i}^{e} u_{i}^{e},
\end{array}\right.
$$

where $B_{i}^{e}$ is the strain field matrix of $i$ th node, $D_{i}^{e}$ is the elastic matrix of $i$ th node, and $u_{i}^{e}$ is the displacement of $i$ th node. The stress and strain of $i$ th node can be derived by the modal displacement of the flexible body. Substituting equation (24) into equation (25), we obtain

$$
\left\{\begin{array}{l}
\varepsilon_{i}^{e}=B_{i}^{e}\left(X_{i}^{e}\right) H_{i}^{e} w=B_{i}^{e}\left(X_{i}^{e}\right) \sum_{j=1}^{H} h_{j i}^{e} w_{j}=\sum_{j=1}^{H} h_{j i}^{e \varepsilon} w_{j}=H_{i}^{e \varepsilon} w, \\
\sigma_{i}^{e}=D_{i}^{e} B_{i}^{e}\left(X_{i}^{e}\right) H_{i}^{e}=D_{i}^{e} B_{i}^{e}\left(X_{i}^{e}\right) \sum_{j=1}^{H} h_{j i}^{e} w_{j}=\sum_{j=1}^{H} h_{j i}^{e \sigma} w_{j}=H_{i}^{e \sigma} w
\end{array}\right.
$$

where $X_{i}^{e}$ is the modal displacement of $i$ th node; $h_{j i}^{e}$ is the $j$ th mode of $i$ th node; $h_{j i}^{e \varepsilon}$ is the strain field generated by the $j$ th mode of $i$ th node; $h_{j i}^{e \sigma}$ is the stress field generated by the $j$ th mode of $i$ th node. Therefore, as long as the modal displacement of each node in the flexible body is obtained, the dynamic stress response of each node in the flexible body can be calculated.

Based on the flexible spring model established in the previous section, the dynamic stress of coil spring in time domain is calculated by modal stress matrix and modal stress recovery method using finite element software and multibody dynamics software. The flexible model and stress cloud diagram of the coil spring are shown in Figure 6.

\section{Dynamic Characteristic Test of Coil Spring}

3.1. Experiment Set-Up. In order to determine the accuracy of the theoretical model and verify the dynamic characteristics of the coil spring, a test bench was set up as shown in Figure 7. It is equipped with an electrohydraulic servo system that allows for an accurate testing at a frequency up to $70 \mathrm{~Hz}$. A controlled sinusoidal movement with varied amplitudes and frequencies was applied as the input. Measurements were carried out by applying such deflections and recording the corresponding forces along the spring axis direction. The lower surface of the spring is fixed, and the sweeping excitation is applied to the upper surface of the spring by a hydraulic servo actuator. Besides, in order to eliminate the influence of inertia force on the actuator tooling, the force sensor is placed in the spring restraint position. By installing the force sensor and displacement sensor in the axial direction of the coil spring, the deformation $s$ and force $F$ of the spring in the test process are measured, and then the relationship between the dynamic stiffness of the coil spring and the excitation frequency is obtained. In addition, strain rosettes are arranged at the position where the stress of the coil spring may be large, and the relationship between the dynamic stress of the spring and the exciting frequency is obtained. In order to ensure the consistency between simulation and test, the boundary conditions of the tested spring are in good agreement with those of simulation.

3.2. Test of Dynamic Stiffness. In order to analyze the dynamic stiffness of the coil spring and verify the accuracy of the equivalent dynamic model of the coil spring, the dynamic stiffness test of the coil spring is carried out, as shown in Figure 8. The lower surface of the spring is fixed, and the sweeping excitation is applied to the upper surface of the spring by a hydraulic servo actuator. The displacement excitation $s$ is as follows:

$$
s=s_{0} \sin (2 \pi f \cdot t)+s_{1},
$$

where $s_{0}$ is the loading amplitude, $f$ is the loading frequency $(1-70 \mathrm{~Hz})$, and $s_{1}$ is the preloading displacement.

The loading displacement of the dynamic characteristic test bench has a certain loss when the excitation frequency is high. In order to accurately reflect the actual displacement excitation of the upper surface of the coil spring, a displacement sensor is installed on the lower surface of the hydraulic actuator. In order to reflect the force response of coil spring under different frequencies, the sweep excitation of $1-70 \mathrm{~Hz}$ is applied to the upper surface of the spring. The sweep excitation spectrum of the upper displacement is shown in Figure 8(b). The transfer force under sweep excitation is shown in Figure 9. It is easy to obtain that the force response of the spring does not change with the frequency in the low frequency range. However, when the excitation frequency is near the resonance frequency of the coil spring, the transfer force of the spring has a significant change.

3.3. Test of Dynamic Stress. In order to analyze the relationship between the dynamic stress and frequency of the coil spring and verify the accuracy of the flexible body model of the coil spring, the dynamic stress test of the coil spring is carried out, as shown 


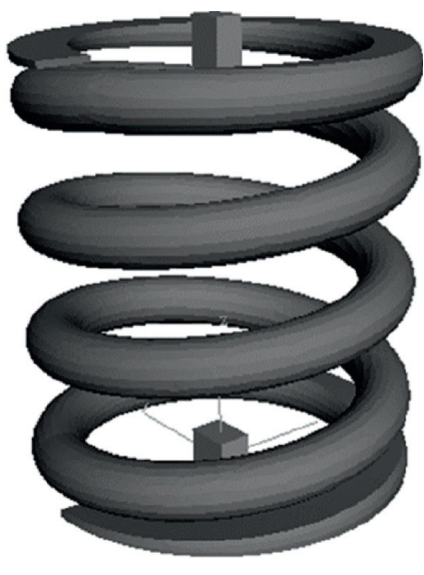

(a)
Equivalent stress

(von mises) $\times 10^{6}$

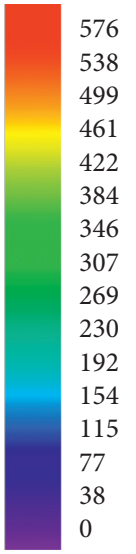

0

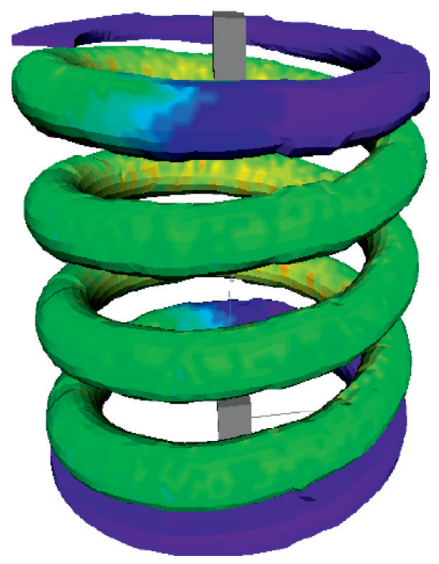

(b)
Displacement $Z$

w.r.t. undeformed $\times 10^{-3}$

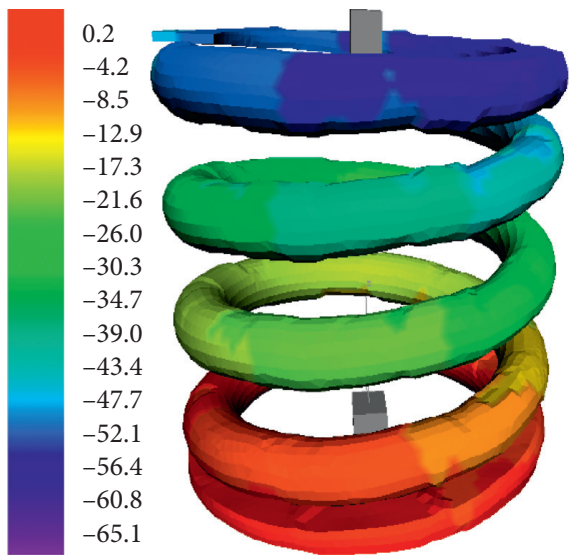

(c)

Figure 6: Simulation of dynamic characteristics. (a) Equivalent model of flexible spring. (b) The cloud diagram of Von Mises stress. (c) The cloud diagram of displacement.

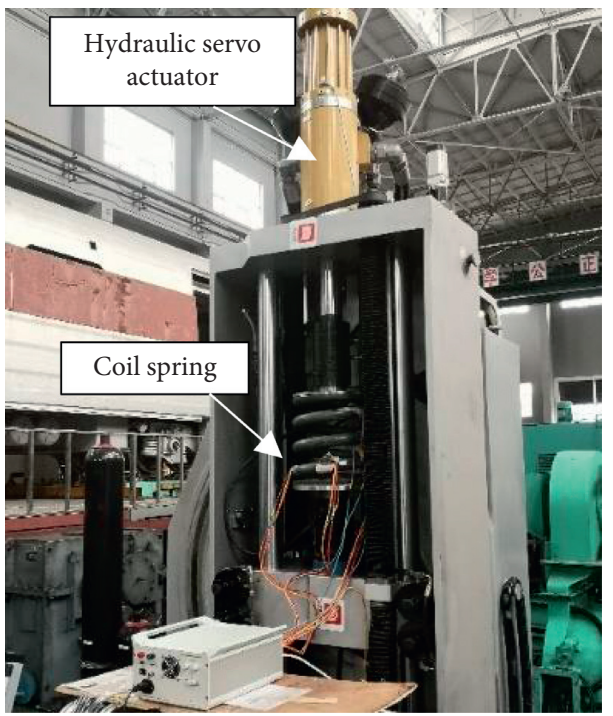

(a)

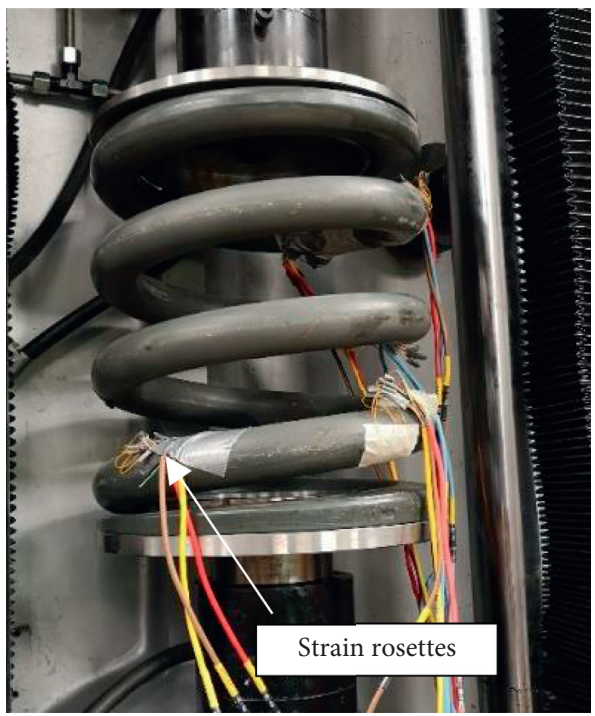

(b)

FIgure 7: Dynamic characteristic test of coil spring. (a) Dynamic characteristic test bench. (b) Installation status of coil spring.

in Figure 7. The constraint and load excitation of the coil spring are consistent with the dynamic stiffness test. The dynamic stress of the coil spring under the sweep excitation is obtained by arranging the stress rosette and stress collector on the coil spring. According to the stress analysis of flexible spring and field test research [12], the area with larger stress of the coil spring appears at the position about 1.2 circles from the lower end, so the measuring points of dynamic stress are arranged at the position about 1.2 circle and middle circle at both ends of the spring, each position is arranged with a three-way strain gauge, and a group of spring has 3 stress measuring points and 9 stress testing channels. The layout of measuring points is shown in Figure 7.
In this test, the adhesive metal strain gauge is used in the spring stress test. The resistance of the strain gauge is proportional to the strain of the equipment. Therefore, the strain of the test sample is directly transmitted to the strain gauge, which causes the linearization of the resistance, and the strain measurement value is obtained through conversion. The direct result of measurement is strain, which is transformed into corresponding stress by using the following equation:

$$
\sigma=E \cdot \varepsilon
$$

where $\sigma$ is the stress value, $\mathcal{\varepsilon}$ is the strain value, and $E$ is the elastic modulus. 


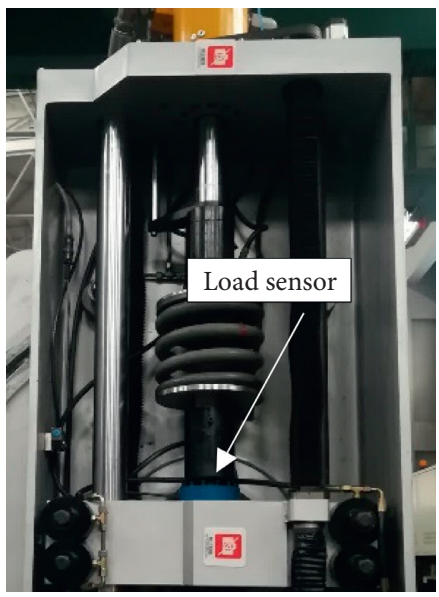

(a)

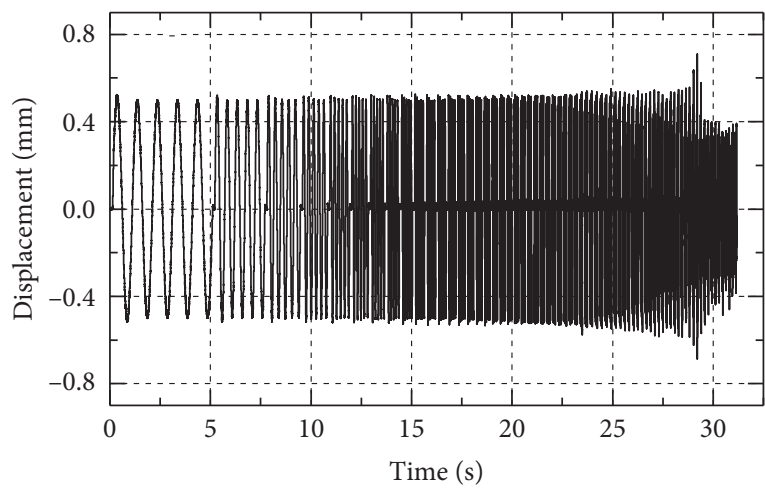

(b)

Figure 8: Dynamic stiffness test of coil spring. (a) Dynamic stiffness test bench. (b) Sweep excitation of displacement.

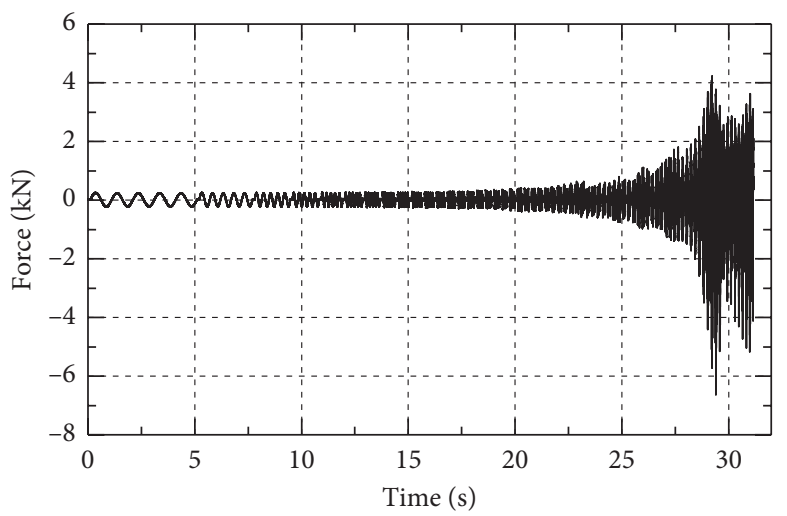

(a)

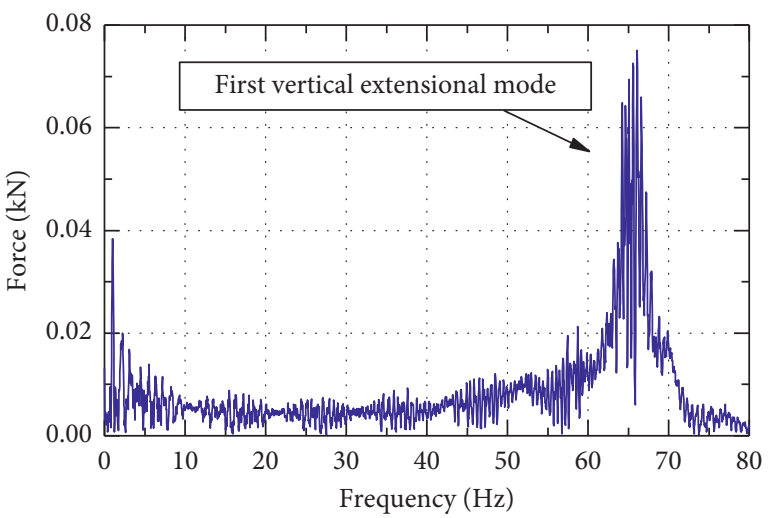

(b)

Figure 9: Dynamic response of transfer force of coil spring. (a) Time-domain response of transferring force. (b) Frequency-domain response of transfer force.

In the field measurement results, the main stress values of strain rosette measuring points in $0^{\circ}, 45^{\circ}$ and $90^{\circ}$ directions are calculated by using the following equations:

$$
\begin{aligned}
& \sigma_{1}=\frac{E}{2}\left[\frac{\left(\varepsilon_{0}+\varepsilon_{90}\right)}{(1-\nu)}+\frac{1}{(1+\nu)} \sqrt{\left(\varepsilon_{0}-\varepsilon_{90}\right)^{2}+\left(2 \varepsilon_{45}-\varepsilon_{0}-\varepsilon_{90}\right)^{2}}\right], \\
& \sigma_{2}=\frac{E}{2}\left[\frac{\left(\varepsilon_{0}+\varepsilon_{90}\right)}{(1-\nu)}-\frac{1}{(1+\nu)} \sqrt{\left(\varepsilon_{0}-\varepsilon_{90}\right)^{2}+\left(2 \varepsilon_{45}-\varepsilon_{0}-\varepsilon_{90}\right)^{2}}\right],
\end{aligned}
$$

where $\sigma_{1}$ and $\sigma_{2}$ are the main stresses, $\nu$ is the Poisson's ratio, and $\varepsilon_{0}, \varepsilon_{45}$, and $\varepsilon_{90}$ are the strain values of strain gauge in $0^{\circ}$, $45^{\circ}$, and $90{ }^{\circ}$ directions, respectively.

After the principal stresses $\sigma_{1}$ and $\sigma_{2}$ are obtained, they are synthesized into the normal form equivalent stress $\sigma_{v}$ :

$$
\sigma_{v}=\sqrt{\frac{1}{2}\left[\sigma_{1}^{2}+\left(\sigma_{1}-\sigma_{2}\right)^{2}+\sigma_{2}^{2}\right]} .
$$

\section{Results and Discussion}

4.1. Comparison of Static Stiffness and Modal Frequency. In order to ensure the consistency of the three models of coil spring with the tested spring, these static stiffnesses of the multi-mass-spring series model, beam element equivalent model, and flexible spring equivalent model are compared with the test results, as shown in Table 5. The above static stiffness is obtained when the excitation frequency is very low. From Table 5, it can be seen that the static stiffness of the three equivalent models differs slightly from the experimental stiffness, which can meet the requirements of the static stiffness of the coil spring in multibody dynamics.

In order to analyze the consistency of dynamic response of equivalent model in multibody dynamic calculation, the constrained modes of three equivalent models and finite element model were compared. According to the installation status of the spring, the two ends of the coil spring are constrained by displacement 
after precompression. The constrained modes of the three equivalent models of the spring are obtained as shown in Table 6.

In the multibody dynamics, the mass and structure of spring can be considered in three equivalent models, so the three models can reflect their modal frequencies and modes. Because the multi-mass-spring series model only has axial stiffness and damping and does not consider the structural heterogeneity, the equivalent model only has the axial expansion mode, which is similar to the finite element model. In Timoshenko beam equivalent model, structural material properties and spirals are defined by beam element, but the nonuniformity of spring structure is not included. Therefore, all modes of coil spring can be covered. The modal frequencies and shape are basically consistent with the results of finite element mode. The equivalent model of flexible spring contains all the material and structural properties of coil spring, which can accurately reflect the modal frequency and shape of spring.

4.2. Comparison and Analysis of Dynamic Stiffness. In multibody dynamics, the equivalent model of coil spring takes into account its mass distribution and geometric structure, so the equivalent model can reflect its modal frequency and shape. When the frequency of the external excitation is close to the spring modal frequency, the coil spring will have abnormal vibration. Significant changes in displacement and force response will lead to significant changes in spring stiffness. In order to calculate the dynamic stiffness of the equivalent spring model, the point stiffness $K_{p}$ and the transfer stiffness $K_{t}$ of the coil spring are defined according to the load-displacement relationship between the free surface and the constrained surface of the coil spring:

$$
\left\{\begin{array}{l}
K_{p}=\frac{F_{b}}{x_{b}}, \\
K_{t}=\frac{F_{t}}{x_{b}},
\end{array}\right.
$$

where $F_{b}$ and $F_{t}$ are, respectively, the upper surface force and the lower surface transfer force of the spring, and $x_{b}$ is the displacement response of the upper surface of the spring.

The time-domain response and frequency-domain response of the reaction force at the lower end of the spring are obtained by applying the sine sweep excitation to the equivalent model of the coil spring and the test piece, as shown in Figure 10. Because the displacement excitation of the test has displacement loss in the high frequency stage, and the cut-off frequency of the test bench is $70 \mathrm{~Hz}$, the simulation analysis frequency is $180 \mathrm{~Hz}$, so the comparative analysis is only for the stiffness rather than the reaction force.

From Figure 10, it can be seen that the reaction force of the coil spring increases abnormally near the frequency of expansion mode. From equation (30), the relationship between the transfer stiffness of the coil spring and the frequency can be obtained. The comparison of stiffness among three equivalent models, common linear spring models, and test results is shown in Figure 11.

The comparison of dynamic stiffness between equivalent model and test results is shown in Figure 11. It is easy to obtain the fact that, compared with the spring linear model, the three equivalent models can reflect the dynamic stiffness varying with frequency. When the external excitation frequency is close to the natural mode frequency of the coil spring, the spring stiffness changes dramatically. Due to the limitation of the dynamic test bench, the maximum external loading frequency is $70 \mathrm{~Hz}$. The resonance effect of coil spring is confirmed in this frequency range. As the multimass-spring series model and Timoshenko-beam model have not considered the structural heterogeneity including the change of pitch and cross section at both ends of spring, the results of stiffness between simulation and test have some differences in modal frequency and amplitude, but the difference is not significant. The flexible spring is able to reflect all of the material and structural characteristics of the coil spring. Compared with the stiffness of the above mentioned models, the dynamic stiffness of the flexible spring has a better agreement with the test values. The results show that the flexible spring can better reflect the vibration characteristics of the coil spring in the multibody dynamic calculation.

4.3. Comparison and Analysis of Dynamic Stress. Under the excitation of sweep frequency, the dynamic stress of the coil spring changes obviously because of the sudden change of the transmitted force in the resonance region. Figure 12 shows the equivalent stress response at the position about 1.2 circle and middle circle of coil spring under the excitation of sweep frequency $(0-70 \mathrm{~Hz})$. It can be seen that the equivalent stress of coil spring near the first extensional frequency is abnormally larger than that in nonresonance area. Compared with the test results, the simulated dynamic stress is completely consistent in frequency and approximate in stress amplitude. Therefore, the flexible body model of coil spring can reflect the change of spring stress in resonance environment.

It can also be seen from the test and simulation that the stress of the coil spring at the position of 1.2 circle is obviously greater than that of the middle circle of the spring. Therefore, it should be noted that the coil spring breaks at the position of 1.2 circle during the actual operation of the coil spring. 
TABle 5: Comparison of the static stiffness.

\begin{tabular}{lcccc}
\hline Order & Test result & Mass-spring series & Timoshenko-beam & Flexible spring \\
\hline Stiffness & 0.475 & 0.4517 & 0.4482 & 0.467 \\
Relation error & - & $4.91 \%$ & $5.64 \%$ & $1.68 \%$ \\
\hline
\end{tabular}

TABle 6: The constrained modes of the coil spring.

\begin{tabular}{|c|c|c|c|c|c|c|c|c|}
\hline \multirow{2}{*}{ Order } & \multicolumn{2}{|c|}{ The finite element model } & \multicolumn{2}{|c|}{ Spring-mass series model } & \multicolumn{2}{|c|}{ Timoshenko-beam model } & \multicolumn{2}{|c|}{ Flexible spring model } \\
\hline & Frequency & Movement mode & Frequency & Movement mode & Frequency & Movement mode & Frequency & Movement mode \\
\hline 1 & 66.1 & Expansion & 65.8 & Expansion & 66.8 & Expansion & 66.1 & Expansion \\
\hline 2 & 70.7 & Twist & 129.2 & Expansion & 71.8 & Twist & 70.6 & Twist \\
\hline 3 & 88.9 & Bending & 187.4 & Expansion & 84.1 & Bending & 88.7 & Bending \\
\hline 4 & 89.7 & Bending & 237.7 & Expansion & 88.5 & Bending & 89.4 & Bending \\
\hline 5 & 117.7 & Expansion & 247.7 & Expansion & 122.9 & Bending & 117.3 & Bending \\
\hline 6 & 122.9 & Twist & 277.2 & Expansion & 127.5 & Expansion & 122.3 & Expansion \\
\hline 7 & 128.8 & Bending & 301.9 & Expansion & 137.1 & Bending & 128.2 & Bending \\
\hline 8 & 133.5 & Bending & 302.4 & Expansion & 144.2 & Twist & 132.7 & Bending \\
\hline
\end{tabular}

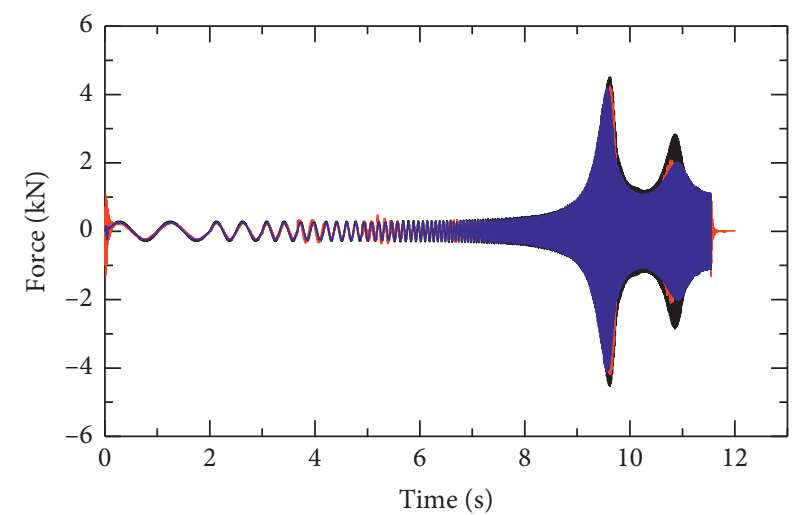

Timoshenko-beam spring
The flexible spring
The spring-mass series

(a)

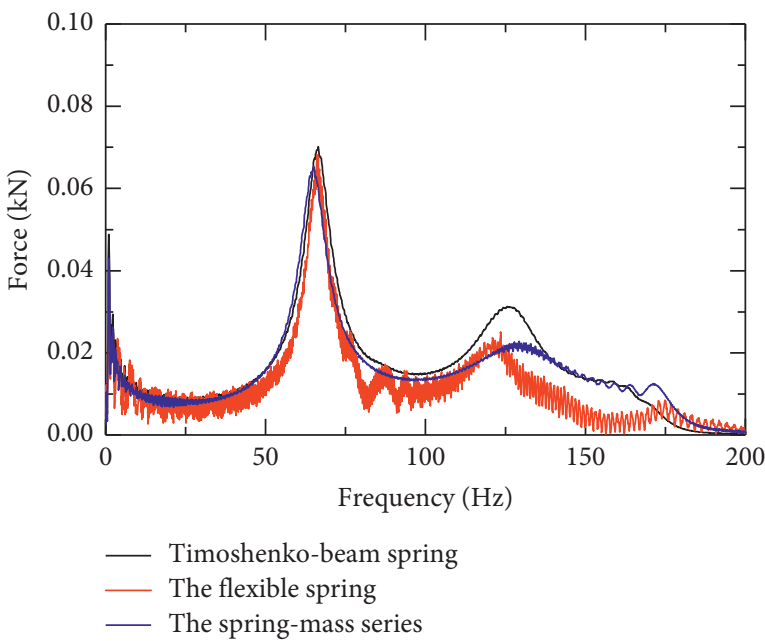

(b)

FIgURE 10: Comparison of reaction forces of three equivalent models. (a) Time domain comparison. (b) Frequency domain comparison.

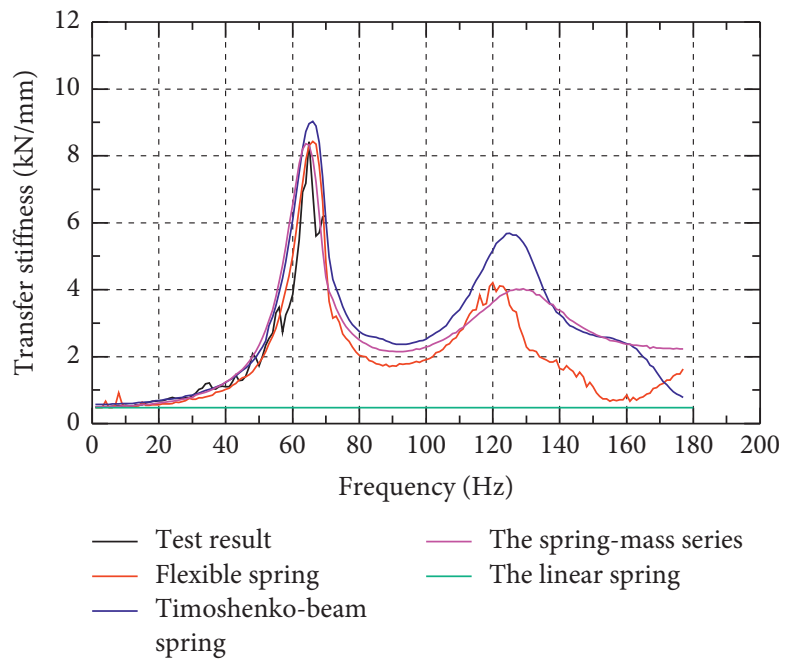

Figure 11: Comparison of dynamic stiffness between equivalent models and test. 


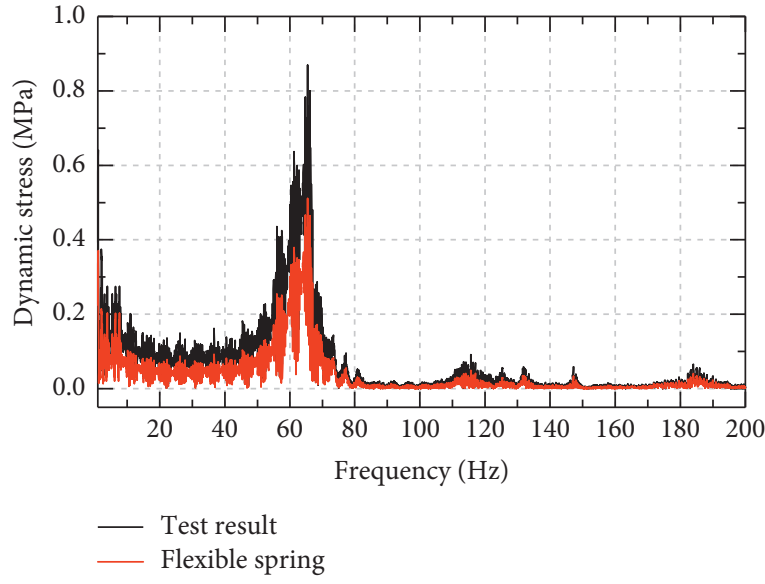

(a)

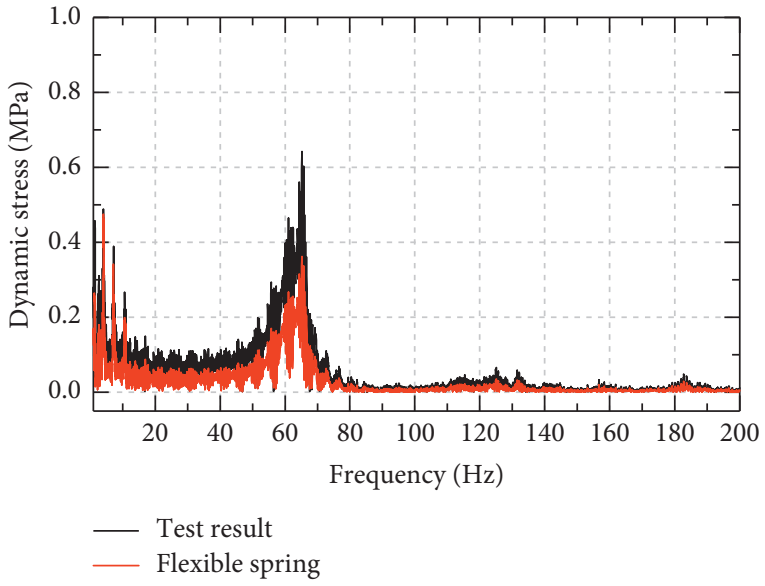

(b)

Figure 12: Comparison of dynamic stress between simulation and experiment. (a) 1.2 circle positions of spring. (b) Middle circle positions of spring.

\section{Conclusions}

In this study, in order to consider the dynamic characteristics of coil springs in multibody dynamics calculation, three equivalent dynamic models of multi-mass-spring series, Timoshenko beam, and flexible spring are proposed. The comparative analysis leads to the following conclusions:

(1) Three equivalent models can reflect the nonlinear relationship of spring dynamic response (force or displacement) with exciting frequency. The multimass-spring series model only considers the axial vibration mode of the spring axis, so the external excitation can only excite the nonlinear characteristics of the spring axial response with frequency.

(2) Compared with the test results of dynamic characteristics of the coil spring, the flexible spring equivalent model can represent the relationship between dynamic stiffness and frequency more accurately than the spring-mass series model and the beam element model. It can also reflect the relationship between dynamic stress and exciting frequency of the coil spring. It can be concluded that the dynamic stiffness and stress of coil spring increase significantly in the resonance region.

(3) The flexible spring equivalent model can not only reflect the frequency-dependent characteristics of dynamic stiffness in vehicle simulation, but also obtain the frequency-dependent characteristics of dynamic stress of spring structure. This will provide an effective way for vehicle dynamics simulation and vibration fatigue analysis of coil spring.

\section{Data Availability}

All data included in this study are available upon request to the corresponding author.

\section{Conflicts of Interest}

The authors declare no conflicts of interest with respect to the research, authorship, and/or publication of this article.

\section{Acknowledgments}

This research was supported by the Independent Research Project of State Key Laboratory of Traction Power (2018TPL_T04), the Youth Program of National Natural Science Foundation of China (51805450), the Youth Program of National Natural Science Foundation of China (51805450) and the National Key R\&D Program of China (2016YFB120404, 2018YFB1201701, and 2018YFE0201401).

\section{References}

[1] S. Bruni, J. Vinolas, M. Berg, O. Polach, and S. Stichel, "Modelling of suspension components in a rail vehicle dynamics context," Vehicle System Dynamics, vol. 49, no. 7, pp. 1021-1072, 2011.

[2] L. Mazzola and M. Berg, "Secondary suspension of railway vehicles - air spring modelling: performance and critical issues," Proceedings of the Institution of Mechanical Engineers, Part F: Journal of Rail and Rapid Transit, vol. 228, no. 3, pp. 225-241, 2014.

[3] M. R. Chi, H. X. Gao, and W. H. Zhang, "Frequency-dependent characteristics of air spring," Journal of Southwest Jiaotong University, vol. 51, no. 2, pp. 236-243, 2016.

[4] S. Bruni and A. Collina, "Modelling the viscoelastic behaviour of elastomeric components: an application to the simulation of train-track interaction," Vehicle System Dynamics, vol. 34, no. 4, pp. 283-301, 2000.

[5] J. Sun, M. Chi, W. Cai, and X. Jin, "Numerical investigation into the critical speed and frequency of the hunting motion in railway vehicle system," Mathematical Problems in Engineering, vol. 2019, Article ID 7163732, 15 pages, 2019.

[6] A. Alonso, J. G. Giménez, and E. Gomez, "Yaw damper modelling and its influence on railway dynamic stability," Vehicle System Dynamics, vol. 49, no. 9, pp. 1367-1387, 2011. 
[7] J. Lee and D. J. Thompson, "Dynamic stiffness formulation, free vibration and wave motion of helical springs," Journal of Sound \& Vibration, vol. 239, no. 2, pp. 297-320, 2011.

[8] K. P. Kumar, S. P. Kumar, and G. Guru Mahesh, "Static analysis of a primary suspension spring used in locomotive," International Journal of Mechanical Engineering and Robotics Research, vol. 2, no. 4, pp. 430-436, 2013.

[9] S. K. Das, N. K. Mukhopadhyay, B. R. Kumar, and D. K. Bhattacharya, "Failure analysis of a passenger car coil spring," Engineering Failure Analysis, vol. 14, no. 1, pp. 158-163, 2007.

[10] C. Zhou, M. R. Chi, Z. F. Wen, and X. W. Wu, "An investigation of abnormal vibration-induced coil spring failure in metro vehicles," Engineering Failure Analysis, vol. 108, Article ID 104238, 2020.

[11] J. Zhang, Z. H. Qi, and Y. P. Zhuo, "Stiffness analysis of helix spring using exact geometric beam element," Engineering Mechanics, vol. 37, no. 2, pp. 16-22, 2020.

[12] W. Sun, J. Zhou, D. Thompson, and D. Gong, "Vertical random vibration analysis of vehicle-track coupled system using Green's function method," Vehicle System Dynamics, vol. 52, no. 3, pp. 362-389, 2014.

[13] W. Sun, D. Thompson, and J. Zhou, "The influence of vehicletrack dynamic coupling on the fatigue failure of coil springs within the primary suspension of metro vehicles," Vehicle System Dynamics, vol. 12, pp. 1-17, 2019.

[14] C. X. Fu and X. L. Wang, "Wave theory modeling of vehicle vibration and its frequency response analysis of helical spring suspension," Chinese Journal of Mechanical Engineering, vol. 41 , no. 5, pp. 54-59, 2015.

[15] H. Liu and W. H. Zhang, "Frequency variety analysis and equivalent algorithm of metal spring stiffness," Journal of Traffic and Transportation Engineering, vol. 7, no. 5, pp. 24-27, 2007.

[16] M. J. Zhou, H. Wang, and B. Wang, "Ultrahigh cycle fatigue damage mechanisms of high strength 50CrVA spring steel regulated by heat treatment process," Chinese Journal of Materials Research, vol. 30, no. 12, pp. 955-960, 2016.

[17] Y. Yan, F. Zhao, and Y. H. Ren, "Failure analysis on spring used in high-speed railway locomotive," Hot Working Technology, vol. 41, no. 22, pp. 232-234, 2012.

[18] V. Yildirim, "Investigation of parameters affecting free vibration frequency of helical springs," International Journal for Numerical Methods in Engineering, vol. 39, no. 1, pp. 99-114, 1996.

[19] V. Yildirim, "Expressions for predicting fundamental natural frequencies of non-cylindrical helical springs," Journal of Sound and Vibration, vol. 252, no. 3, pp. 479-491, 2002.

[20] L. E. Becker, G. G. Chassie, and W. L. Cleghorn, "On the natural frequencies of helical compression springs," International Journal of Mechanical Sciences, vol. 44, no. 4, pp. 825-841, 2002.

[21] F. F. Calim, "Dynamic analysis of composite coil springs of arbitrary shape," Composites Part B: Engineering, vol. 40, no. 8, pp. 741-757, 2009.

[22] W. J. Sun and D. Gong, "Influence of dynamic stiffness of primary suspension on vehicle-track coupled vibration," Journal of Vibration and Shock, vol. 34, no. 5, pp. 49-55, 2015.

[23] X. Q. Li, Z. X. Deng, and C. B. Li, "Substructure normal modes selection method for component mode synthesis," Journal of Southwest Jiaotong University, vol. 49, no. 1, pp. 173-178, 2014.

[24] A. H. Liao, X. Huang, and Y. Fang, "Fatigue analysis for bogie frame on rigid-flexible coupling simulation," Journal of Vibration, Measurement \& Diagnosis, vol. 37, no. 2, pp. 394-395, 2017. 